\title{
Anapole Moment and Other Constraints on the Strangeness Conserving Hadronic Weak Interaction
}

\author{
W. C. Haxton* and C.-P. Liu ${ }^{\dagger}$ \\ Institute for Nuclear Theory, Box 351550, University of Washington, Seattle, Washington 98195-1550 \\ and Department of Physics, University of Washington, Seattle, Washington 98195-1550 \\ M. J. Ramsey-Musolf \\ Kellogg Radiation Laboratory, California Institute of Technology, Pasadena, California 91125 \\ and Department of Physics, University of Connecticut, Storrs, Connecticut 06269 \\ and Theory Group, Thomas Jefferson National Accelerator Facility, \\ Newport News, Virginia 23606
}

(Received 10 January 2001)

\begin{abstract}
Standard analyses of low-energy $N N$ and nuclear parity-violating observables have been based on a $\pi-, \rho$-, and $\omega$-exchange model capable of describing all five independent $s-p$ partial waves. Here a parallel analysis is performed for the one-body, exchange-current, and nuclear polarization contributions to the anapole moments of ${ }^{133} \mathrm{Cs}$ and ${ }^{205} \mathrm{Tl}$. The resulting constraints are not consistent, though there remains some degree of uncertainty in the nuclear structure analysis of the atomic moments.
\end{abstract}

DOI: $10.1103 /$ PhysRevLett.86.5247

Nuclei and nucleon-nucleon scattering are the only experimentally tractable systems in which to study the flavorconserving hadronic weak interaction, where neutral current effects arise. This interaction can be isolated, despite the presence of much larger strong and electromagnetic effects, because of the accompanying parity violation. The long-term goal of the field is to learn how standard-model quark-boson couplings give rise to long-range weak forces between nucleons [1-3].

Several precise and interpretable measurements of parity nonconservation (PNC) in nuclear systems have been made. These include the longitudinal analyzing power $A_{z}$ for $\vec{p}+p$ at 13.6 and $45 \mathrm{MeV}, A_{z}$ for $\vec{p}+\alpha$ at $46 \mathrm{MeV}$, the circular polarization $P_{\gamma}$ of the $\gamma$ ray emitted from the $1081 \mathrm{keV}$ state in ${ }^{18} \mathrm{~F}$, and $A_{\gamma}$ for the decay of the $110 \mathrm{keV}$ state in polarized ${ }^{19} \mathrm{~F}$. An analysis [2] of these results, which have been in hand for some time, suggests that the isoscalar PNC NN interaction is comparable to or somewhat stronger than the "best value" suggested theoretically, while the isovector PNC $N N$ interaction is significantly weaker, an isospin anomaly superficially reminiscent of the $\Delta I=1 / 2$ rule in strangeness-changing decays.

After a considerable wait, several new PNC measurements have become available or are expected soon. Recently the Colorado group [4] measured, for the first time, a nuclear anapole moment - the PNC axial coupling of a photon to the nucleus in its ground state - by determining the hyperfine dependence of atomic parity violation. A significant limit on the anapole moment of another nucleus, ${ }^{205} \mathrm{Tl}$, has also been obtained [5]. Preliminary results [6] for the $\vec{p}+p A_{z}(221 \mathrm{MeV})$ are now available, and experiments on the PNC spin rotation of polarized slow neu-
PACS numbers: 21.30.Fe, 13.75.Cs, 24.70.+s, 24.80.+y

trons in liquid helium and on $A_{\gamma}$ in $n+p \rightarrow d+\gamma$ are underway.

The primary obstacle to an analysis in which the new PNC constraints are combined with older results is the difficulty of treating anapole moments with a comparable degree of sophistication. The theoretical framework for $N N$ and nuclear observables is a $\pi-, \rho-$, and $\omega$-exchange model involving six weak meson-nucleon couplings $f_{\pi}$, $h_{\rho}^{0}, h_{\rho}^{1}, h_{\rho}^{2}, h_{\omega}^{0}$, and $h_{\omega}^{1}$, as defined by Desplanques, Donoghue, and Holstein (DDH) [1] (so the sign of $f_{\pi}$ differs from that of Ref. [7]). For low-momentum phenomena this framework is quite general, describing the five independent $s-p$ amplitudes and the separate long-range $\pi$ contribution to those amplitudes.

The older PNC results involve systems that are either amenable to exact potential-model calculations, or can be "calibrated" experimentally [2]. Calculations account for the full two-body PNC potential and the effects of short-range correlations on the potential. In contrast, most anapole moment investigations have been evaluated in the extreme single-particle (s.p.) limit employing effective one-body potentials. The only calculation employing a modern strong effective interaction in combination with a PNC two-body potential was limited to the effects of $f_{\pi}$ [7]. Here that calculation (for ${ }^{133} \mathrm{Cs}$ ) is extended to the full potential and then repeated for ${ }^{205} \mathrm{Tl}$. We then examine the consistency of these and other constraints on the weak potential.

The anapole moment, the E1 coupling of a virtual photon to an elementary particle induced by PNC, was introduced by Zel'dovich [8]. Flambaum, Khriplovich, and Sushkov [9] then argued that anapole moments of heavy atoms might be sufficiently enhanced to be measurable. 
Electrons in an atom experience a weak contact interaction with the nucleus of the form

$$
H_{W}=\frac{G_{F}}{\sqrt{2}} \kappa \vec{\alpha} \cdot \vec{I} \rho(r),
$$

where $\vec{I}$ and $\rho(r)$ are the nuclear spin and density and $\vec{\alpha}$ is the Dirac matrix operating on the electrons. (Note that $\kappa$ differs from the definition of $[9,10]$.) From the hyperfine dependence of the atomic PNC signals in ${ }^{133} \mathrm{Cs}$ (as extracted by Flambaum and Murray [10]) and ${ }^{205} \mathrm{Tl}$ [5] one finds

$$
\begin{aligned}
\kappa\left({ }^{133} \mathrm{Cs}\right) & =0.112 \pm 0.016 \\
\kappa\left({ }^{205} \mathrm{Tl}\right) & =0.293 \pm 0.400
\end{aligned}
$$

One contribution to $\kappa$ originates from $Z_{0}$ exchange with axial coupling to the nucleus

$$
\kappa_{Z_{0}}=-\frac{g_{A}}{2}\left(1-4 \sin ^{2} \theta_{W}\right) \frac{\left\langle I\left\|\sum_{i=1}^{A} \sigma(i) \tau_{3}(i)\right\| I\right\rangle}{\langle I \| \hat{I}|| I\rangle},
$$

where $g_{A}=1.26$ is the axial vector coupling, $\sin ^{2} \theta_{W}=$ 0.223 , and || denotes a matrix element reduced in angular momentum. The reduced matrix element of $\hat{I}$ is $\sqrt{I(I+1)(2 I+1)}$. The Gamow-Teller matrix elements, taken from shell model (SM) studies described below, are $-2.305\left({ }^{133} \mathrm{Cs}\right)$ and $2.282\left({ }^{205} \mathrm{Tl}\right)$, close to the proton $1 g_{7 / 2}$ and $3 s_{1 / 2}$ s.p. values of -2.494 and 2.449 . Thus the predicted $\kappa_{Z_{0}}$ are 0.0140 and -0.127 , respectively. Note that one-loop standard model electroweak radiative corrections will modify these results somewhat [11].

A second contribution to $\kappa$ is generated by the combined effects of the usual coherent $Z_{0}$ coupling to the nucleus (vector coupling, proportional to the nuclear weak charge $\left.Q_{W}\right)$ and the magnetic hyperfine interaction [12]. From the measured nuclear weak charge and magnetic moment Bouchiat and Piketty [13] find

$$
\begin{aligned}
\kappa_{Q_{W}}\left({ }^{133} \mathrm{Cs}\right) & =0.0078, \\
\kappa_{Q_{W}}\left({ }^{205} \mathrm{Tl}\right) & =0.044 .
\end{aligned}
$$

Thus the experimental values for the anapole contributions to $\kappa$ are obtained by subtracting the results of Eqs. (3) and (4) from Eq. (2), yielding

$$
\begin{aligned}
\kappa_{\text {anapole }}\left({ }^{133} \mathrm{Cs}\right) & =0.090 \pm 0.016, \\
\kappa_{\text {anapole }}\left({ }^{205} \mathrm{Tl}\right) & =0.376 \pm 0.400 .
\end{aligned}
$$

These values can then be related to the corresponding nuclear anapole moments by

$$
\kappa_{\text {anapole }}=\frac{4 \pi \alpha \sqrt{2}}{M_{N}^{2} G_{F}} \frac{\left\langle I\left\|\hat{A}_{1}\right\| I\right\rangle / e}{\langle I|| \hat{I} \| I\rangle},
$$

where the anapole operator $\hat{A}_{1 \lambda}$ can be written, via the extended Siegert's theorem, in a form where all components of the current that are constrained by current conservation are explicitly removed. This yields [7]

$$
\begin{aligned}
\hat{A}_{1 \lambda}= & -\frac{M_{N}^{2}}{9} \int d \vec{r} r^{2} \\
& \times\left[\hat{j}_{1 \lambda}^{e m}(\vec{r})+\sqrt{2 \pi}\left[Y_{2}\left(\Omega_{r}\right) \otimes \hat{j}_{1}^{e m}(\vec{r})\right]_{1 \lambda}\right] .
\end{aligned}
$$

We now consider the various contributions to this operator.

(a) Nucleon anapole moment.-The one-body PNC electromagnetic current is obtained from, for example, loop diagrams involving one strong and one weak mesonnucleon coupling. The $E 1$ projection of this PNC current yields the one-body contribution to Eq. (7)

$$
\hat{A}_{1 \lambda}^{\text {one-body }}=\sum_{i=1}^{A}\left[a_{s}(0)+a_{v}(0) \tau_{3}(i)\right] \sigma_{1 \lambda}(i) .
$$

In our earlier work [7] only the pion contribution to $a_{s}(0)$ and $a_{v}(0)$ was included, yielding a result proportional to $e f_{\pi} g_{\pi N N}$, where $g_{\pi N N}$ is the strong coupling. The isoscalar coupling $a_{s}(0)$ then dominates. This was extended recently to the full set of one-loop contributions involving the DDH vector meson PNC couplings, using the framework of heavy baryon chiral perturbation theory and retaining contributions through $O\left(1 / \Lambda_{\chi}^{2}\right)$, where $\Lambda_{\chi}=4 \pi F_{\pi} \sim 1 \mathrm{GeV}$ is the scale of chiral symmetry breaking [11]. The addition of the heavy mesons greatly enhances $a_{v}(0)$ and thus the overall nucleon anapole moment. An evaluation with DDH best value couplings yields $a_{v}(0) \sim 7 a_{s}(0)$. Folding the resulting expressions with our SM matrix elements $\left(\left\langle I|| \sum_{i=1}^{A} \sigma(i) \| I\right\rangle=-2.372\right.$ and 2.532 for $\mathrm{Cs}$ and $\mathrm{Tl}$, respectively) yields the results in Table I.

(b) Exchange currents.-Insertion of the $N \bar{N}$ pair and transition currents, where the meson exchange involves a

TABLE I. Decomposition of the SM estimates of the anapole matrix element $\left\langle I|| A_{1} \| I\right\rangle / e$ into its weak coupling contributions.

\begin{tabular}{ccrrrrrr}
\hline \hline Nucleus & Source & \multicolumn{1}{c}{$f_{\pi}$} & \multicolumn{1}{c}{$h_{\rho}^{0}$} & \multicolumn{1}{c}{$h_{\rho}^{1}$} & \multicolumn{1}{c}{$h_{\rho}^{2}$} & \multicolumn{1}{c}{$h_{\omega}^{0}$} & \multicolumn{1}{c}{$h_{\omega}^{1}$} \\
\hline${ }^{133} \mathrm{Cs}$ & One-body & 0.59 & 0.87 & 0.90 & 0.36 & 0.28 & 0.29 \\
& Ex. cur. & 8.58 & 0.02 & 0.11 & 0.06 & -0.57 & -0.57 \\
& Polariz. & 51.57 & -16.67 & -4.88 & -0.06 & -9.79 & -4.59 \\
& Total & 60.74 & -15.78 & -3.87 & 0.36 & -10.09 & -4.87 \\
${ }^{205} \mathrm{Tl}$ & One-body & -0.63 & -0.86 & -0.96 & -0.35 & -0.29 & -0.29 \\
& Ex. cur. & -3.54 & -0.01 & -0.06 & -0.03 & 0.28 & 0.28 \\
& Polariz. & -13.86 & 4.63 & 1.34 & 0.08 & 2.77 & 1.27 \\
& Total & -18.03 & 3.76 & 0.33 & -0.30 & 2.76 & 1.26 \\
\hline \hline
\end{tabular}


PNC coupling on one nucleon and a strong coupling to the second, into Eq. (7) produces a two-body PNC anapole operator. The only previous estimate [7] of contributions of this type was restricted to pions. The extension to include the $\rho$ and $\omega$ PNC couplings is a formidable task requiring evaluation of the $\rho$ and $\omega$ pair currents and the $\rho \rho \gamma$ and $\rho \pi \gamma$ currents. An initial simple Fermi gas calculation showed that the $\rho \rho \gamma, \rho \pi \gamma$, and the component of the $\omega$ pair current where the photon and PNC $\omega$ couplings are on different nucleon legs are negligible, well below $1 \%$ of the dominant $\pi$ currents. The remaining important heavymeson terms were evaluated using the two-body density matrices from our large-basis SM calculations. The exchange current totals are given in Table I. It is clear that the $\pi$ contribution continues to dominate. This work is described in considerable detail in Ref. [14].

(c) Nuclear polarization contribution.-The nuclear polarization contribution to the anapole moment is given by

$$
\sum_{n} \frac{\left\langle I|| \hat{A}_{1}^{e m} \| n\right\rangle\left\langle n\left|H^{\mathrm{PNC}}\right| I\right\rangle}{E_{g s}-E_{n}}+\text { H.c., }
$$

where $\hat{A}_{1}^{e m}$ is obtained from the ordinary electromagnetic current operator, $|I\rangle$ is a ground state of good parity, $H^{\mathrm{PNC}}$ is the PNC $N N$ interaction, and the sum extends over a complete set of nuclear states $n$ of angular momentum $I$ and opposite parity.

The canonical SM space for ${ }^{133} \mathrm{Cs}$ is that between the magic shells 50 and $82,1 g_{7 / 2}-2 d_{5 / 2}-1 h_{11 / 2}-3 s_{1 / 2}-2 d_{3 / 2}$. Calculations were performed with protons restricted to the first two of these shells and neutron holes to the last three, producing a $m$-scheme basis of about 200000 . Two effective interactions designed for the ${ }^{132} \mathrm{Sn}$ region were employed, the Baldrige-Vary potential used in Ref. [7] and one developed recently by the Strasbourg group [15]. As the results are very similar [14], we quote only the former here. ${ }^{205} \mathrm{Tl}$ is described as a proton hole in the orbits immediately below the $Z=82$ closed shell $\left(3 s_{1 / 2}-2 d_{3 / 2}-2 d_{5 / 2}\right)$ coupled to two neutron holes in valence neutron space between magic numbers 126 and 82 $\left(3 p_{1 / 2}-2 f_{5 / 2}-3 p_{3 / 2}-1 i_{13 / 2}-2 f_{7 / 2}-1 h_{9 / 2}\right)$. A Serber-Yukawa force was diagonalized in this space.

The summation over a complete set of intermediate states in such spaces is impractical either directly or by the summation-of-moments method discussed in Ref. [7]. Instead we complete the sum by closure after replacing $1 / E_{n}$ by an average value $\langle 1 / E\rangle$. For our SM spaces the resulting product of $A_{1}^{e m}$ and $H^{\mathrm{PNC}}$ contracts to a two-body operator, so that only the two-body ground state density matrix is needed.

The closure approximation is useful if we can identify $\langle 1 / E\rangle$ with something measurable, such as the distribution of E1 strength in the corresponding nucleus. To investigate the systematics we completed a series of exact calculations in $1 p$ - and light $2 s 1 d$-shell nuclei $\left({ }^{7} \mathrm{Li},{ }^{11} \mathrm{~B},{ }^{17,19,21} \mathrm{~F}\right.$, ${ }^{21,23} \mathrm{Na}$ ), determining the ground states from full $0 \hbar \omega$ diagonalizations. After performing the summations (by Lanczos moments methods [7]) over the $1 \hbar \omega$ spaces, the dimensions of which range up to $\sim 0.5 \mathrm{M}$, we found that the anapole and E1 closure energies tracked each other very well, provided one takes into account the three isospins contributing to $H^{\mathrm{PNC}}$ (see [14]). Measured as a fraction of the $1 / E$-weighted giant dipole average excitation energy, which is $\langle 1 / E\rangle^{-1} \sim(22-26) \mathrm{MeV}$ for these nuclei, the appropriate effective energies for the anapole closure approximation are $0.604 \pm 0.056\left(h_{\rho}^{0}, h_{\omega}^{0}\right), 0.899 \pm 0.090$ $\left(f_{\pi}\right)$, and $1.28 \pm 0.14\left(h_{\rho}^{2}\right)$. The larger $\langle 1 / E\rangle$ for $h_{\rho}^{0}$ and $h_{\omega}^{0}$ enhances the isoscalar contribution to the anapole polarizability. The small variation in $\langle 1 / E\rangle$, once the isospin dependence is recognized, supports the notion that we can connect the closure result to the true polarization sum. From the known $E 1$ distribution [16] in ${ }^{133} \mathrm{Cs}$ we then determine $T=0,1,2$ closure energies of 9.5, 14.1, and $20.2 \mathrm{MeV}$, respectively. That is, we fix these as $0.6,0.9$, and 1.28 of the $E 1$ closure energy evaluated from the experimental dipole distribution. The corresponding ${ }^{205} \mathrm{Tl}$ values are $8.7,12.9$, and $18.5 \mathrm{MeV}$. The resulting polarization contributions are given in Table I.

A summary of PNC constraints is presented in Table II and Fig. 1. Although the PNC parameter space is six-dimensional, two coupling constant combinations, $f_{\pi^{-}}-0.12 h_{\rho}^{1}-0.18 h_{\omega}^{1}$ and $h_{\rho}^{0}+0.7 h_{\omega}^{0}$, dominate the observables. We include the results for $A_{z}^{p p}$ at 13.6, 45, and $221 \mathrm{MeV}, A_{z}^{p \alpha}$ at $46 \mathrm{MeV}, P_{\gamma}\left({ }^{18} \mathrm{~F}\right), A_{\gamma}\left({ }^{19} \mathrm{~F}\right)$, and the $\mathrm{Cs}$ and $\mathrm{Tl}$ anapole results. We do not include $P_{\gamma}\left({ }^{21} \mathrm{Ne}\right)$ because of the arguments given in Ref. [2]. The $1 \sigma$ error bands of Fig. 1 are generated from the experimental

TABLE II. PNC observables and corresponding theoretical predictions, decomposed into the designated weak-coupling combinations.

\begin{tabular}{cccccrrr}
\hline \hline Observable & Exp. $\left(\times 10^{7}\right)$ & $f_{\pi^{-}}-0.12 h_{\rho}^{1}-0.18 h_{\omega}^{1}$ & $h_{\rho}^{0}+0.7 h_{\omega}^{0}$ & \multicolumn{1}{c}{$h_{\rho}^{1}$} & \multicolumn{1}{c}{$h_{\rho}^{2}$} & \multicolumn{1}{c}{$h_{\omega}^{0}$} & $h_{\omega}^{1}$ \\
\hline$A_{z}^{p p}(13.6)$ & $-0.93 \pm 0.21$ & & 0.043 & 0.043 & 0.017 & 0.009 & 0.039 \\
$A_{z}^{p p}(45)$ & $-1.57 \pm 0.23$ & & 0.079 & 0.079 & 0.032 & 0.018 & 0.073 \\
$A_{z}^{p p}(221)$ & Prelim. & & -0.030 & -0.030 & -0.012 & 0.021 & \\
$A_{z}^{p \alpha}(46)$ & $-3.34 \pm 0.93$ & -0.340 & 0.140 & 0.006 & & -0.039 & -0.002 \\
$P_{\gamma}\left({ }^{18} \mathrm{~F}\right)$ & $1200 \pm 3860$ & 4385 & & 34 & & -4.5 & -0.1 \\
$A_{\gamma}\left({ }^{19} \mathrm{~F}\right)$ & $-740 \pm 190$ & -94.2 & 34.1 & -1.1 & & -4.1 \\
$\left\langle\| A_{1}||\right\rangle / e, \mathrm{Cs}$ & $800 \pm 140$ & 60.7 & -15.8 & 3.4 & 0.4 & 1.0 & 6.1 \\
$\left\langle\left\|A_{1}\right\|\right\rangle / e, \mathrm{Tl}$ & $370 \pm 390$ & -18.0 & 3.8 & -1.8 & -0.3 & 0.1 & -2.0 \\
\hline \hline
\end{tabular}






FIG. 1. Constraints on the PNC meson couplings $\left(\times 10^{7}\right)$ that follow from the results in Table II. The error bands are one standard deviation.

uncertainties, broadened somewhat by allowing uncorrelated variations in the parameters in the last four columns of Table II over the DDH broad "reasonable ranges." Before the anapole results are included, the indicated solution is a small $f_{\pi}$ and an isoscalar coupling somewhat larger, but consistent with, the DDH best value, $-\left(h_{\rho}^{0}+0.7 h_{\omega}^{0}\right)^{\mathrm{DDH} \text { b.v. } \sim 12.7 . \quad \text { The anapole results }}$ agree poorly with the indicated solution, as well as with each other. Although the $\mathrm{Tl}$ measurement is consistent with zero, it favors a positive anapole moment, while the theory prediction is decidedly negative, given existing PNC constraints. The Cs result tests a combination of PNC couplings quite similar to those measured in $A_{\gamma}\left({ }^{19} \mathrm{~F}\right)$ and in $A_{z}^{p \alpha}$, but favors larger values.

This discrepancy - the Cs anapole moment requiring larger PNC couplings - is surprising. The first criticism of the theory would be that the SM calculations are still too limited, not generating the proper quenching of operators such as $\sigma \tau_{3}$ that are known to be sensitive to core polarization effects. There is evidence, in the case of $\mathrm{Tl}$ where the odd proton is identified with the $l=03 s_{1 / 2}$ orbital, that this is the case: the SM predicts a spindominated magnetic moment of $2.59 \mu_{N}$, improved from the s.p. value $2.79 \mu_{N}$ but well above the experimental value $1.64 \mu_{N}$. Indeed, in Ref. [13] similar arguments were used to invoke quenching factors for the s.p. anapole moment predictions. Further quenching, of course, will exacerbate the discrepancies.

Our numerical results for Cs are consistent with those of Flambaum and Murray [10], who extract from the anapole moment an $f_{\pi}$ about twice the DDH best value, $f_{\pi}^{\text {DDH b.v. }} \sim 4.6$, and point out that theory can accommo- date this. (The DDH reasonable range is $0-11.4$, in units of $10^{-7}$.) However, this ignores $P_{\gamma}\left({ }^{18} \mathrm{~F}\right)$, a measurement that has been performed by five groups. The resulting constraint is almost devoid of theoretical uncertainty

$$
-0.6 \lesssim f_{\pi}-0.11 h_{\rho}^{1}-0.19 h_{\omega}^{1} \lesssim 1.2 .
$$

Allowing $h_{\rho}^{1}$ and $h_{\omega}^{1}$ to vary throughout their DDH reasonable ranges, one finds $-1.0 \lesssim f_{\pi} \lesssim 1.1$, clearly ruling out $f_{\pi} \sim 9$. Figure 1 illustrates this, as well as the additional tension between $\mathrm{Cs}, p+\alpha$, and $A_{\gamma}\left({ }^{19} \mathrm{~F}\right)$.

In summary there appears to be a puzzle to sort out. A resolution is needed because our understanding of $V(e)-A(N)$ interactions also affects the interpretation of experiments like SAMPLE [17], where a similar discrepancy between theory and experiment exists.

This work was supported in part by the U.S. Department of Energy and by the National Science Foundation.

*Email address: haxton@phys.washington.edu

${ }^{\dagger}$ Email address: cpliu@u.washington.edu

\#Email address: mjrm@phys.uconn.edu

[1] B. Desplanques, J. F. Donoghue, and B. R. Holstein, Ann. Phys. (N.Y.) 124, 449 (1980).

[2] E. G. Adelberger and W. C. Haxton, Annu. Rev. Nucl. Part. Sci. 35, 501 (1985).

[3] W. Haeberli and B. R. Holstein, in Symmetries and Fundamental Interactions in Nuclei, edited by W. C. Haxton and E. Henley (World Scientific, Singapore, 1995), p. 17.

[4] C. S. Wood et al., Science 275, 1759 (1997).

[5] P. A. Vetter et al., Phys. Rev. Lett. 74, 2658 (1995).

[6] S. Page and W. van Oers (private communications); (to be published).

[7] W. C. Haxton, E. M. Henley, and M. J. Musolf, Phys. Rev. Lett. 63, 949 (1989); W. C. Haxton, Science 275, 1753 (1997).

[8] Ya. B. Zel'dovich, Sov. Phys. JETP 6, 1184 (1957).

[9] V. V. Flambaum and I. B. Khriplovich, Sov. Phys. JETP 52, 835 (1980); V. V. Flambaum, I. B. Khriplovich, and O.P. Sushkov, Phys. Lett. B 146, 367 (1984).

[10] V. V. Flambaum and D. W. Murray, Phys. Rev. C 56, 1641 (1997); also see W. S. Wilburn and J.D. Bowman, Phys. Rev. C 57, 3425 (1998).

[11] M. J. Musolf and B. R. Holstein, Phys. Lett. B 242, 461 (1990); Phys. Rev. D 43, 2956 (1991); S.-L. Zhu, S. J. Puglia, B. R. Holstein, and M. J. Ramsey-Musolf, Phys. Rev. D 62, 033008 (2000).

[12] V. V. Flambaum and I. B. Khriplovich, Sov. Phys. JETP 62, 872 (1985); M. G. Kozlov, Phys. Lett. A 130, 426 (1988).

[13] C. Bouchiat and C. A. Piketty, Z. Phys. C 49, 91 (1991); Phys. Lett. B 269, 195 (1991).

[14] W. C. Haxton, C.-P. Liu, and M. J. Ramsey-Musolf (to be published).

[15] F. Nowacki (private communication).

[16] B. L. Berman et al., Phys. Rev. 177, 1745 (1969).

[17] R. Hasty et al., Science 290, 2117 (2000). 